\title{
Saisie directe sur le site de l'information pluviographique
}

\author{
Direct acquisition of rainfall data on site
}

\author{
R. Ferrero et G. Galea \\ Divisions Calcul et Hydrologie - CEMAGREF - Antony
}

Réflexions sur la codification de l'information pluviographique du bassin versant de l'Orgeval

Divers aspects méthodologiques développés à la Division Hydrologie du CEMAGREF d'Antony, nécessitent la mise sur support traitable des observations réalisées sur le bassin versant expérimental de l'Orgeval. En particulier, la codification de l'information d'un réseau dense de pluviographes Filotechnica (21 postes sur $100 \mathrm{~km}^{2}$ ) à rotation hebdomadaire représente un travail non négligeable. Pour cela nous utilisons la procédure éprouvée et opérationnelle de dépouillement semiautomatique (Procédure Pluie 1) mise au point par le Service de l'Hydraulique et le CEMAGREF et dont la phase première consiste à préparer, puis ensuite à digitaliser, les pluviogrammes. Ces procédures passent par l'utilisation d'une table de lecture couplée à un TEKTRONIX 4051 (16 K) permettant de critiquer et de saisir le fichier ainsi constitué sur cassette magnétique. Cette phase de préparation et de digitalisation des pluviogrammes. Bien que très rigoureuse sur le plan de la méthode, n'en demeure pas moins lourde et relativement subjective dans la préparation (dépouillement à intensité constante, position des points "cerises", choix de la base de temps, précision des cochages, horaires de contrôle de la dérive des horloges mécaniques, etc.) et la digitalisation (précision du pointé, fixation des diagrammes, etc.).

Cette subjectivité est accrue par des contraintes d'exploitation inévitables comme par exemple la faible vitesse de rotation des tambours ( $2 \mathrm{~mm} /$ heure).

Les logiciels développés compte tenu des diverses sources d'erreurs, sont suffisamment contraignants pour assurer une bonne qualité du fichier $P(t)$. Cependant cette indispensable valorisation du fichier $P(t)$ a l'inconvénient d'augmenter considérablement la durée d'obtention d'un fichier $P(t)$ opérationnel, ce qui, pour notre part, freine les études que l'on désirerait mener.

Aussi, dans un souci de plus grande efficacité, nous nous sommes orientés vers un codage sur cassette magnétique de l'information pluviographique directement sur le site, grâce à des codeurs autonomes. Compte tenu de l'ancienneté de notre réseau (1962), de sa finalité et des moyens dont nous disposons tant financiers que techniques ou en personnel qualifié, nous avons porté notre choix sur une petite station autonome semi-automatique. Les essais effectués antérieurement par EDF-DTG et la Météorologie Nationale (Trappes) nous ont guidé dans ce choix. Ce dernier a abouti à l'appareil AS 40/01 réalisé par Automatisme et Système.

\section{Le codeur enregistreur AS 40/01}

Le codeur enregistreur AS/40.01 est fabriqué par la Société Automatisme et Système : 204, Avenue Marx Dormoy à Montrouge (92120). Tél. 656.08.20.

\section{Description (Fig. I)}

L'enregistreur magnétique de précipitations se présente sous la forme d'un coffret polyester renforcé verre armé de $20 \mathrm{~cm}$ de hauteur, $33 \mathrm{~cm}$ de largeur et $22 \mathrm{~cm}$ de profondeur. Son poids est de $7 \mathrm{~kg}$. C'est un multi-voies (6) qui admet des entrées digitales (impulsions) et analogiques. La technologie utilisée est une technologie CMOS assurant une grande immunité contre les bruits de toutes sortes et ne nécessitant qu'une très faible consommation.

Sa configuration de base assez souple permet une adaptation des 6 entrées aux besoins de l'utilisateur suivant les paramètres qu'il désire enregistrer. Pour 


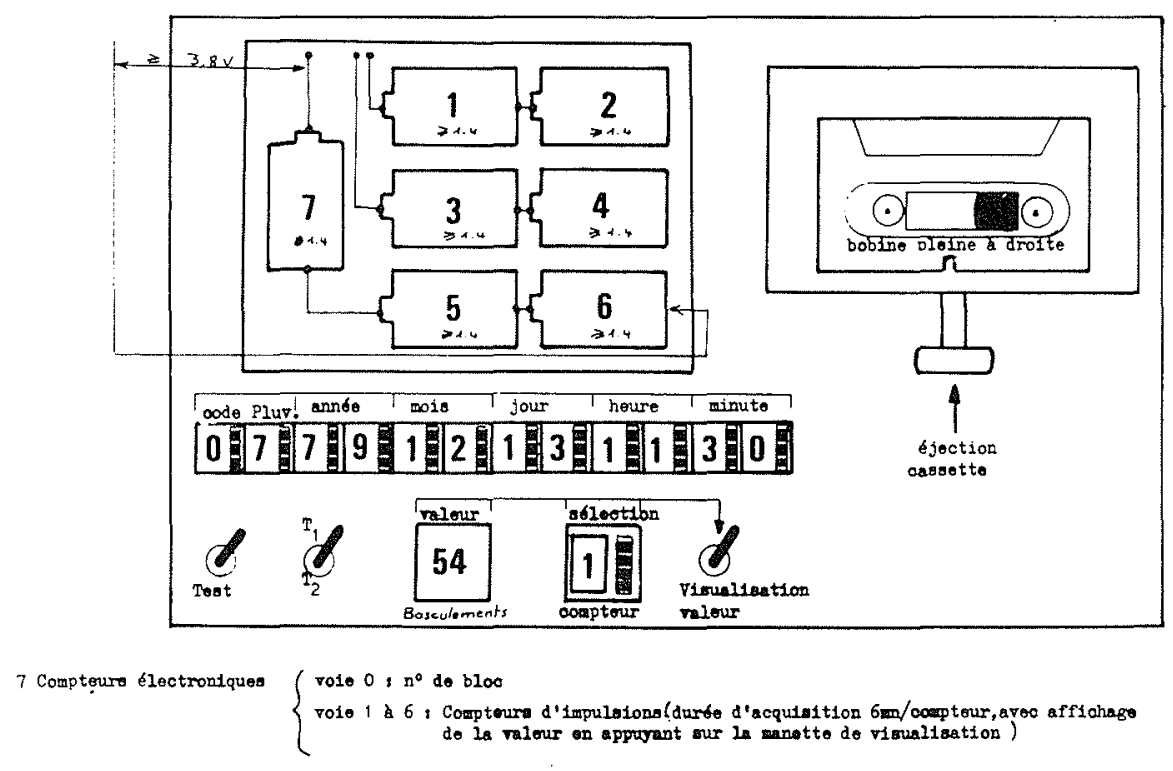

Figure 1 - Platine du codeur AS/40-01.

notre part, désireux d'enregistrer l'information pluviographique, nous avons opté pour une configuration qui, compte tenu des normes d'enregistrement ECMA 34 , nous assure la plus grande autonomie possible et la plus grande sécurité au niveau de la lecture des cassettes magnétiques. C'est-à-dire la configuration : mono-voie à entrée digitale à laquelle sont affectés 6 compteurs électroniques strappés entre eux ; pas de scrutation de $6 \mathrm{~min}$; une longueur de bloc d'enregistrement de 26 octets.

Sa mise en œuvre a été simplifiée à l'extrême ; toutes les commandes se font à partir d'une platine horizontale accessible après avoir soulevé le couvercle.

Cette platine comporte principalement :

- Un boittier renfermant 7 piles alcalines de 1,5 V, qui procurent l'énergie nécessaire au moteur d'entraînement de la cassette et à l'électronique de base ; celleci étant alimentée en continu par 3 de ces piles ;

- Un témoin lumineux (vert ou rouge) indiquant une tension normale ou anormale de fonctionnement;

- 12 roues codeuses, qui permettent de créer un label d'identification du contenu de la cassette ;

- Un bouton poussoir marqué TEST, qui permet de vérifier le bon fonctionnement de l'électronique interne $\mathrm{du}$ codeur sur les 6 compteurs électroniques. L'acquisition est de 512 impulsions (horloge à quartz) pour un pas de scrutation de $2 \mathrm{~s}$. Le maintien du bouton TEST, associé à un dispositif de visualisation et de sélection du numéro du compteur, permet de visualiser pour chaque compteur les 512 impulsions. Le relâchement $\mathrm{du}$ bouton provoque le défilement de la cassette pendant $20 \mathrm{~s}$, l'écriture du label d'identification au bout de ces 20 s et le début de l'acquisition au pas de temps présélectionné ;

- Un dispositif de visualisation servant, soit à l'affichage du contenu d'un compteur ( 7 compteurs à 3 digits modulo 999), soit à l'affichage d'une valeur de TEST ; - Une roue "codeuse", servant à sélectionner le numéro du compteur dont l'affichage du contenu est demandé, y compris le compteur 0 (compteur de blocs) ;
- Un bouton poussoir commandant l'affichage ;

- Un dérouleur de cassette comportant la mécanique de défilement, la tête d'écriture, ainsi que le mécanisme d'éjection et d'encliquetage.

\section{Principe de fonctionnement}

\section{Adaptation d'un système transducteur aux pluvio- graphes Filotecnica}

Le pluviomètre transducteur que nous trouvons sur le marché est le pluviomètre transducteur du type R 013030 A de chez Précis-Mécanique, et qui est beaucoup utilisé par la Météorologie Nationale.

Pour le réseau un peu ancien du bassin versant de l'Orgeval les pluviographes Filotecnica (8) ont été équipés d'un système transducteur représenté par un interrupteur magnétique "REED" placé dans la partie avant du cache des augets, orthogonalement à l'axe pivot des augets et par un barreau aimanté fixé sur

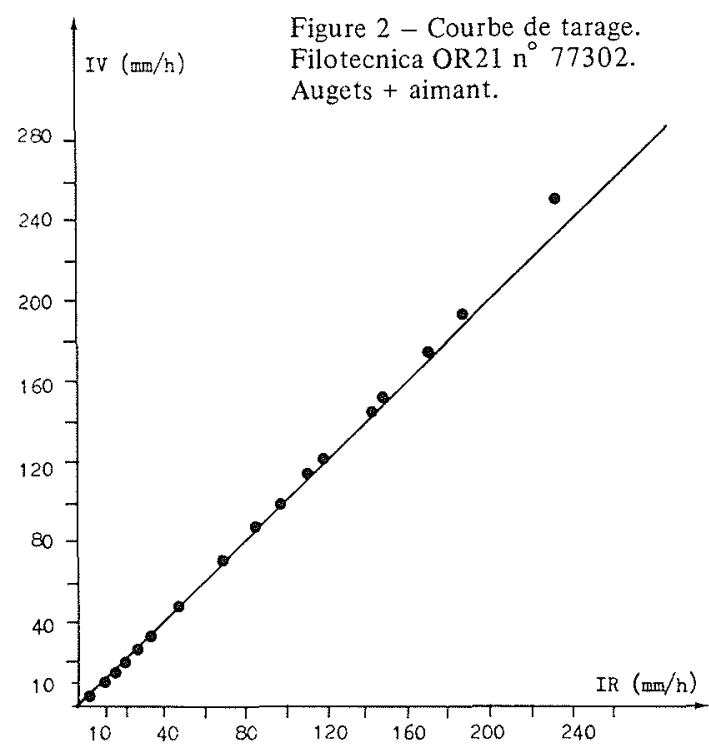


l'axe pivot des augets. Cette surcharge des augets n'a pas modifié la courbe théorique de fonctionnement du pluviographe (Fig. 2). Les tests de vieillissement des interrupteurs "REED" ont été négatifs.

\section{L'acquisition au niveau du codeur}

A chaque basculement d'auget $(0,2 \mathrm{~mm})$, l'interrupteur "REED" soumis à un champ magnétique ferme le circuit impulsionnel qui est alors parcouru par un courant de $50 \mu \mathrm{A}$ et ceci se traduit, au niveau de l'enregistreur, par l'acquisition numérique correspondant à une impulsion électrique. Ces impulsions sont ensuite cumulées dans des compteurs électroniques (6) pendant le pas de scrutation sélectionné $(6 \mathrm{~min})$ et au bout d'une durée égale à $6 \min \times 6=36 \mathrm{~min}$ leur contenu est transféré en bloc sur le support magnétique. La logique de transfert incrémente au fur et à mesure ces différents blocs d'une unité.

\section{Installation des codeurs $A S / 40.01$ sur le site}

Il faut éviter de placer l'appareil près d'une source possible de foudre (pylône, transformateur), de phénomènes électromagnétiques (ligne), ou de bruits susceptibles d'agir sur le fonctionnement magnétique.

Le codeur sera posé sur le fond de l'abri, au-dessous du seau, en s'assurant qu'il est bien positionné horizontalement afin que son étanchéité aux projections d'eau soit parfaite (Fig. 3).

\section{Considérations générales sur l'exploitation du codeur}

On enregistre simultanément la pluie sur diagramme et sur support magnétique (cassettes). Le diagramme permettra de valoriser le fichier cassette si des anomalies surviennent au niveau du capteur (ex. mise en charge de l'entonnoir, etc.).

\section{Changement de bande}

Il faudra faire attention, lors du changement de bande, à ne pas générer des impulsions (basculement d'auget) qui, bien entendu, seraient prises en compte sur la cassette. Il suffit pour cela, aussi bien pour le changement de bande que pour le réglage en amplitude de l'enregistrement graphique, de débrancher une borne du circuit impulsionnel.

Lors de chaque tournée du réseau, on vérifie le bon fonctionnement du codeur en procédant aux contrôles suivants :

\section{Le voyant des piles:}

Le voyant des piles doit être impérativement de couleur verte; signe que l'enregistreur fonctionne sous une tension normale; sinon il faut rapidement les remplacer après avoir éjecté la cassette en cours d'acquisition (tension coupée).

On doit aussi effectuer une mesure de la tension aux bornes de chaque pile qui devra être $\geqslant 1,4$ volts et vérifier que le circuit d'alimentation de l'électronique n'est pas inférieur à 3,8 volts (Fig. 1).

En effet, la baisse de tension des piles (par températures très basses : $-10^{\circ} \mathrm{C},-15^{\circ} \mathrm{C}$ ) est la cause d'un

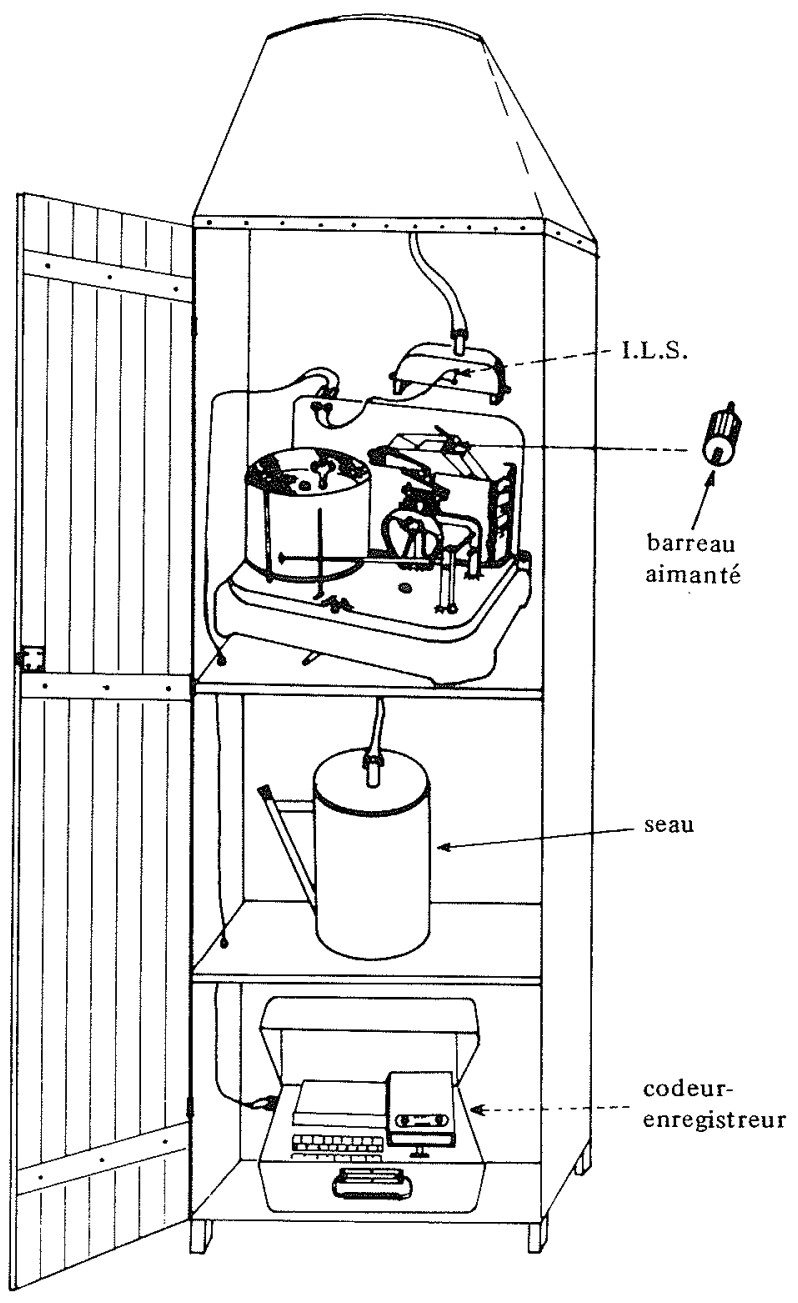

Figure 3 - Vue d'ensemble : capteur et codeur.

certain nombre d'ennuis techniques qui ont été mis en évidence au Centre Technique et du Matériel (CTM) de la Météorologie Nationale à Trappes. La baisse de tension des piles provoque, en particulier, des variations de vitesse de défilement de la bande, incompatibles avec les exigences des codes d'enregistrement à densité fixe (ECMA 34). Elle a aussi pour conséquence de faire varier le courant d'excitation de la tête d'écriture et par suite de provoquer des pertes d'information par défaut de magnétisation.

A la limite, si la baisse de tension devient trop importante, on aura un "gap" de fin de fichier jusqu'à la fin de la cassette.

\section{Remplacement du deshydratant}

Sous l'action combinée de la température et de l'humidité, on a des condensations sur les platines d'enregistrement qui peuvent provoquer des arrêts du système d'entraînement (givrage des parties mobiles), des courants de fuite provoquant des inscriptions aberrantes, etc. Donc, il faut introduire un produit deshydratant (du silicagel, par exemple). Après chaque intervention entraînant l'ouverture du boîtier, le corps desséchant doit être remplacé. 


\section{Cassettes d'enregistrements et piles à utiliser}

Pour limiter le nombre d'ennuis techniques pouvant survenir à basses températures (généralement ces codeurs fonctionnent bien entre 0 et $40^{\circ} \mathrm{C}$ ) on utilisera :

\section{Cassettes}

Des cassettes spéciales, dites "à basses températures" (par exemple, cassette ITC., réf. H 300). En effet, les cassettes ordinaires restant plusieurs heures sous tension à l'arrêt subissent des déformations qui ne peuvent être résorbées par la suite. Lors de la relecture de ces cassettes, il y a une perte d'information constatée (par le CTM) du fait que la distance support-tête de lecture devient momentanément incorrecte.

\section{Piles}

La source d'alimentation du codeur sera assurée par 7 piles alcalines convenant pour toute installation autonome fonctionnant dans un domaine compris entre $-15^{\circ} \mathrm{C}$ et $+40^{\circ} \mathrm{C}$. Ces piles ont, à volume égal, une capacité environ trois fois supérieure et un meilleur comportement aux faibles températures que les piles à électrolyte salin.

\section{Autonomie}

La configuration adoptée permet une autonomie énergétique d'environ 3 mois.

Cette configuration est celle qui permet la plus grande autonomie sur le plan énergétique, car le moteur d'entrainement de la cassette (principale source de consommation : $200 \mathrm{~mA}$ pendant 1 à $2 / 10 \mathrm{~s}$ ) ne sera sollicité que toutes les $36 \min (6 \min \times 6)$.

\section{La cassette magnétique}

L'enregistrement est conforme aux critères des normes ECMA 34, qui permettent d'assurer une compatibilité absolue entre différents systèmes. Chaque enregistrement comprend un bloc d'identification défini à partir des 12 roues codeuses (Fig. 1) et $n$ blocs de données.

Le codage des données est un codage ASCII ; ASCII étant un code de transmission standard à 8 bits.

Dans la configuration retenue, l'autonomie de la bande magnétique est d'environ 2 mois $1 / 2$.

\section{Considérations générales sur l'exploitation des cassettes}

\section{Fréquence des relevés}

Pour des raisons d'exploitation, les cassettes sont relevées et traduites à la fin de chaque mois.

\section{Précautions particulières}

- à la pose de la cassette, prévoir au préalable quelques tours d'enroulement de la bande magnétique afin d'être sûr que l'acquisition commencera bien après le "sticker" (tout ce qui sera avant ne pourra être décodé);

- à l'éjection de la cassette, le bloc en cours d'acqujsition est perdu, mais cela peut aussi provoquer d'une manière non systématique une erreur de "CRC" c'est-à-dire de contrôle sur le bloc précédent. On enlèvera donc la cassette compte tenu de sa relative grande autonomie à un moment sans pluie, supérieur à la durée de deux blocs ( $36 \mathrm{~min} \times 2=72 \mathrm{~min}$ ), afin de ne pas perdre d'information;

- envoi des cassettes au centre de traitement: les cassettes enregistrées sur le terrain sont envoyées au centre de lecture (à Antony) pour effectuer le traitement informatique :

- un bordereau accompagne chaque cassette et permet d'une part, d'identifier le label de définition saisi sur la cassette et, d'autre part, d'intégrer au traitement informatique les observations de terrain, en particulier les contrôles au seau qui permettent de s'affranchir des anomalies aléatoires dues au fonctionnement hydraulique et mécanique du pluviographe ;

- retour des cassettes sur le terrain : une fois les cassettes dépouillées et le fichier $P(t)$ constitué et vérifié, les cassettes sont effacées, réenroulées et prêtes pour une nouvelle utilisation.

\section{Dérive en temps}

La fonction de synchronisation est assurée par une horloge à quartz de période $32,768 \mathrm{KHz}$ couplée à des circuits diviseurs.

La dérive absolue est actuellement inférieure à la minute pour un mois d'enregistrement.

\section{Traitement Informatique}

Par souci de simplifier et de diminuer les interventions manuelles du personnel technique chargé de l'exploitation des codeurs, on reporte quelques difficultés au niveau des programmes de traitement de ces enregistrements magnétiques. En résumé, l'observateur n'interviendra que pour vérifier la bonne marche du codeur (changer les piles, si nécessaire, la cassette, etc.), et notera sur un bordereau les anomalies constatées au niveau du capteur et du codeur.

\section{Le lecteur de cassette}

Le lecteur de cassette est un appareil spécialisé, de type LDB 4201, qui fonctionne de manière télécommandćc, couplé à un micro-ordinatcur TEKTRONIX $(16 \mathrm{~K})$.

\section{Les programmes}

Le traitement informatique fait appel à deux programmes complémentaires : le programme DCAS (Décodage Cassette) et le programme PCAS (Pluie Cassette) qui sont intégrés dans la programmathèque des traitements de base en hydrologie (Fig. 4).

\section{Le programme DCAS}

Le programme DCAS permet de télécommander par l'intermédiaire du TEKTRONIX les différentes fonctions du lecteur de cassette assurées par microprocesseur comme l'enroulement de la cassette, le positionnement sur le premier bloc, etc. 


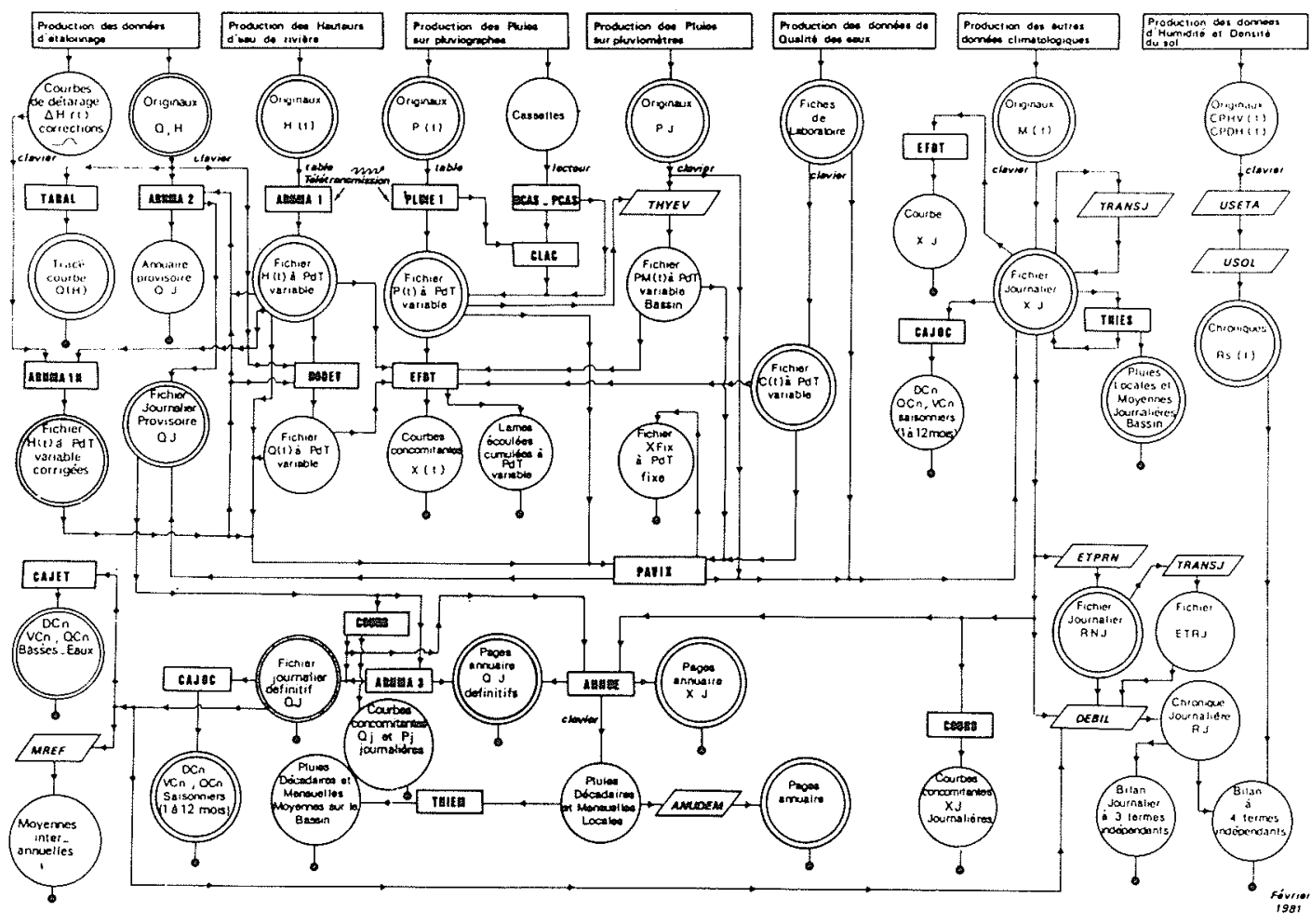

Figure 4 - Schéma de la programmathèque des traitements de base en hydrologie (SH-CEMAGREF-SRAE).

Le temps de décodage de la cassette est assez long, 20 min en moyenne par mois d'enregistrement (1120 blocs de données). Ceci est relativement long, mais se justifie par le fait que le logiciel a été développé dans un esprit "bassin versant expérimental" et non pas "réseau" ; ce logiciel effectue une critique de l'enregistrement pluviographique avec en particulier affichage des anomalies sur l'écran au fur et à mesure du traitement, etc.

La lecture de la cassette ne nécessite la présence de l'opérateur qu'au début et à la fin du traitement; après celui-ci l'opérateur relève les anomalies éventuelles pour ensuite valoriser l'enregistrement pluviographique magnétique.

Cette mini-chaine de traitement et de critique des cassettes est autonome et indépendante d'un gros système (IRIS 80).

\section{Le programme $P C A S$}

Ce logiciel a été développé sur IRIS 80 ; il est donc nécessaire d'utiliser une liaison de transfert du contenu de la cassette TEKTRONIX (ex. TRANSPAC).

Le programme PCAS permet la traduction du fichier en sortie de DCAS (Fig. 5) en pluie cumulée à pas de temps variable $P(t)$ (Fig. 6).

\section{Conclusions}

Depuis environ 2 ans, ce type de codeur-enregistreur conforme à la configuration précédemment décrite répond à nos diverses préoccupations en matière de saisie et de traitement de l'information pluviographique, en ce qui concerne :

- les conditions d'environnement :

$-15^{\circ} \mathrm{C}<\mathrm{T}^{\circ} \mathrm{C}<+40^{\circ} \mathrm{C}$

Lors des périodes de gel, l'enregistrement magnétique est discontinu (blocage à cause du gel), par contre,

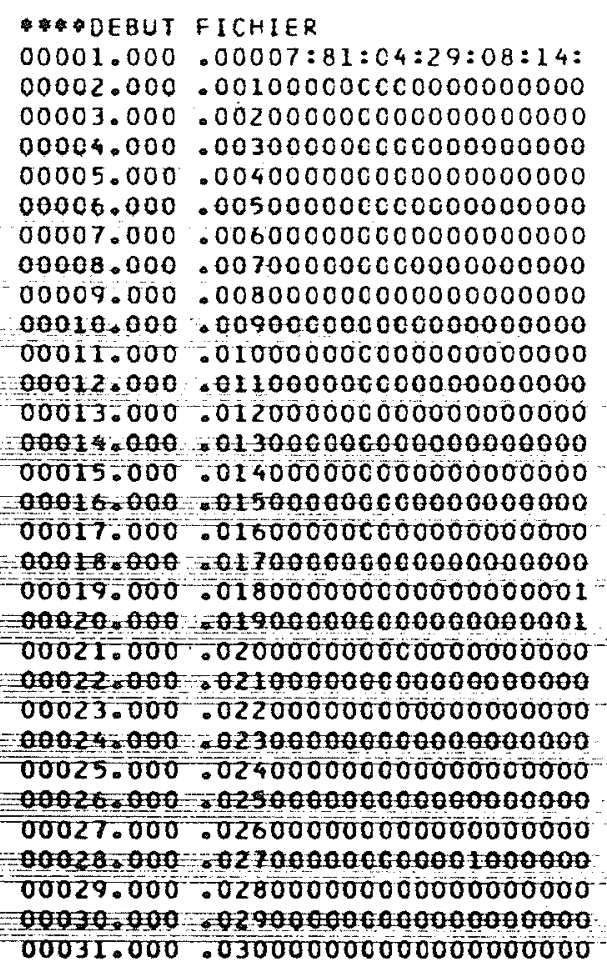

Figure 5 


\begin{tabular}{|c|c|c|c|c|c|c|c|c|c|c|c|c|c|c|c|c|}
\hline 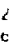 & $\begin{array}{l}77642211 \\
77642211\end{array}$ & $\begin{array}{l}\text { El } \\
\text { El }\end{array}$ & $5 \leqslant . E 4 t$ & c & $95.99 t$ & 0 & $96.00 \mathrm{C}$ & 2 & 96.117 & 2 & st. 121 & 15 & $5 t .275$ & 25 & $i$ & $\begin{array}{l}18 C \\
18 C\end{array}$ \\
\hline e & 77642211 & el & $5 t .2 E 4$ & 14 & $9 t .288$ & 23 & $9 t .29 t$ & 23 & $96.3 \mathrm{CC}$ & 25 & $96.34 t$ & 25 & $5 t .350$ & 27 & 2 & 180 \\
\hline c & $77(4) 211$ & $t 1$ & ऽ E. ¿ $\angle 3$ & 27 & 98.267 & 29 & $10 c .20 \mathrm{c}$ & 29 & 100.264 & 35 & 100.213 & 39 & $1 C C .217$ & 43 & $\bar{z}$ & 180 \\
\hline$t$ & $7704 i 211$ & 61 & $1 C C .<a:$ & 50 & 100.236 & 67 & 100.242 & 69 & $100 . i+t$ & 12 & 100.254 & $7 t$ & 100.754 & $7 t$ & 2 & $18 \mathrm{C}$ \\
\hline & 77042211 & $\varepsilon \mathrm{I}$ & $1 C C . i \leq 5$ & 86 & $100.7 t 3$ & 92 & 100.767 & 152 & 100.731 & 179 & 100.775 & 189 & 160.379 & 195 & 2 & $16 \mathrm{C}$ \\
\hline$t$ & 77042211 & $E 1$ & $\left.I C C_{D}\right) E_{4}$ & 197 & 100.788 & 197 & 100.792 & 199 & $100.75 t$ & 199 & 100.806 & 201 & $1 C C .804$ & $2 \mathrm{Cl}$ & i & $18 \mathrm{C}$ \\
\hline$t$ & $77 C 4 \times 211$ & $t 1$ & $1 C C, E C G$ & $2 \mathrm{G3}$ & 101.042 & 203 & $101.04 t$ & 205 & $103.14 t$ & 205 & $1 C 3.15 \mathrm{C}$ & 207 & 165.217 & 207 & $z$ & 180 \\
\hline 6 & $7764<211$ & EI & 105.221 & 209 & 108.342 & $2 \mathrm{CG}$ & $-4 C .00 C$ & 0 & $-40 . c c 0$ & c & -40.000 & c & $-4 c \cdot c 00$ & 0 & $z$ & $18 \mathrm{C}$ \\
\hline
\end{tabular}

Figure 6

lorsque l'information pluviographique reprend, celle-ci est bien enregistrée et localisée dans le temps;

- l'autonomie : en ce qui concerne la source d'énergie (piles), elle est d'environ 3 mois ; pour ce qui est de la durée d'enregistrement (cassette) elle est d'environ 2 mois $1 / 2$;

- le pas de scrutation : le pas de scrutation (6 min) permet une bonne connaissance de la pluie sur les petits pas de temps ( 7 pas de scrutation de $2 \mathrm{~s}$ à $60 \mathrm{~min})$;

- la dérive en temps : elle est au maximum de quelques minutes par mois (actuellement réduite à moins de $1 \mathrm{~min}$ ), ce qui permet au niveau du capteur et du réseau une bonne synchronisation des évènements pluviométriques;

- la lecture des cassettes : les blocs d'enregistrements très courts (26 octets) diminuent les risques de perte d'information (dus aux contraintes de fonctionnement) au moment de la lecture; le taux d'erreurs, s'il n'est pas négligeable est tout à fait acceptable. La mini-chaîne de traitement (lecteur + TEKTRONIX) est peu performante (20 min pour décoder 1 mois : 1120 blocs) mais convenable dans une optique "bassin versant expérimental".

\section{Avenir des codeurs-enregistreurs à cassettes}

Il faut bien se rendre compte que les systèmes de télétransmissions restent encore assez onéreux et surtout assujettis à un certain nombre de contraintes législatives (radio) ou propres aux gestionnaires de certaines chaines d'acquisition (EDF, Satellites...). Leur généralisation ne pourrait être effective dans l'immédiat et peut-être pas non plus dans la décennie à venir. Dans l'attente, un créneau assez important apparaît pour les codeurs autonomes d'un coût relativement modeste, et dont les récents progrès en ce qui concerne le stockage (sur cassette magnétique, sur cassette à bulles magnétiques, etc.) en font des appareils bien adaptés aux contraintes thermiques de terrain. Cela devrait permettre à beaucoup de stations de passer par un stade économiquement raisonnable de semi-automatisation, en attendant l'éventuelle généralisation de la télétransmission par satellite.

Les alternatives de codeurs autonomes sur autre support que les cassettes (mémoires statiques, etc.) sont pour l'instant trop coûteuses ou de capacité trop limitée (autonomie). 


\section{Discussion}

Président : M. P. BROCHET

Le Président. - Les enregistrements, tels que vous les avez décrits M. GALEA présentent l'avantage d'un traitement plus simple, plus rapide, et plus homogène, que les enregistrements graphiques, et sont beaucoup moins coûteux que les moyens de transmissions.

A la Météorologie Nationale nous avons utilisé des matériels analogues. Outre quelques déboires liés essentiellement à une réalisation imparfaite, ces expérimentations soulèvent certaines difficultés :

- le choix d'une norme permettant un bon enregistrement à basse température. A ce titre, nous préférerons la norme ISO 4349 à la norme ECMA 34 que vous utilisez;

- le temps de dépouillement important pour un fonctionment de réseau. Le Conseil Supérieur de la Météorologie, sous l'impulsion de M. ETIENNE, a recensé les postes pluviométriques jugés fiables. Il y en a environ 5 500. Même si l'on s'en tient au réseau pluviométrique national actuel, le dépouillement de ses 3500 postes nécessiterait environ 1000 heures/mois selon les durées de dépouillement que vous avez indiquées. L'enregistrement magnétique facilite grandement le dépouillement des mesures de hauteurs des précipitations.

M. DUTILLET (Servide de l'Hydraulique - Ministère de l'Agriculture). - L'enregistrement graphique et la digitalisation des diagrammes à la main n'ont encore jamais fait l'objet d'une application en réseau. Il est donc difficile d'en estimer les coûts et il n'est pas prouvé que, lorsque l'on aura tenu compte également du temps de critique des enregistrements sur cassettes, la comparaison financière soit à l'avantage de ces derniers. Néanmoins, cette expérience intéresse tout-à-fait le Ministère de l'Hydraulique.

M. GALEA. - Le temps de dépouillement manuel des diagrammes est long. Le dépouillement des cassettes (20 min pour 1 mois d'observation) ne nécessite la présence de l'opérateur que pour lancer le programme et relever en fin de traitement sur l'écran de visualisation, les anomalies rencontrées lors de la lecture, dont on tiendra compte ultérieurement pour valoriser le fichier obtenu.

M. DUTILLET. - Selon nos estimations, dans le cas des hauteurs d'eau en rivière (où l'on a l'expérience de 10 ans sur 1000 sites dépouillés), pour être compétitif avec la digitalisation manuelle, il faudrait que l'enregistreur et le codeur coûtent de l'ordre de $5000 \mathrm{~F}$. De tels équipements sont à l'étude chez de petits constructeurs.

M. GALEA. - Le capteur lui-même est à ce prix.Pour tous ces matériels. un effet d'échelle permettrait d'abaisser les coûts unitaires, mais nos travaux n'ont eu comme ambition que de répondre à nos propres besoins.

M. CHERON (Service Technique de l'Urbanisme). - L'enregistrement graphique permet un contrôle visuel par les gens de terrain qui constitue un aspect essentiel de leur motivation.

Par ailleurs, dans la course actuelle aux nouvelles technologies, sans cesse dépassées à leur tour, il faut savoir vivre et exploiter du matériel ancien. Conservons les enregistrements graphiques, c'est une sécurité essentielle.

$M$. BEDIOT (Agence Financière de Bassin "SeineNormandie"). - Il faut tout de même rappeler que le dépouillement des pluviogrammes est un travail fastidieux, à tel point qu'actuellement, seules sont dépouillées en France les deux séries de Montpellier Bel Air et Paris Montsouris. En l'absence de personnel et de matériel pour faire ce travail considérable à l'échelle de la France, une technique d'enregistrement sur le site serait utile. Tant qu'un fonds suffisant de données pluviographiques n'est pas constitué, il est difficile d'en évaluer l'étendue des utilisations possibles et d'en dresser le bilan économique.

M. OBLED. - Quelle précision peut-on atteindre dans ce dépouillement et à quel pas de temps obtient-on des données fíables?

M. GALEA. - Le degré de précision est lié à des contraintes d'exploitation du réseau. Pour un enregistrement graphique, tout dépend de la vitesse de déroulement des enregistreurs. En passant de $2 \mathrm{~mm} / \mathrm{h}$ à $65 \mathrm{~mm} / \mathrm{h}$, on peut espérer une meilleure connaissance de la structure fine de la pluie. Le pas d'intégration minimal est la demi-heure, pour des vitesses d'avancement de $2 \mathrm{~mm} / \mathrm{h}$.

Pour les enregistrements sur cassette magnétique on peut descendre à quelques seondes si le besoin s'en fait sentir, pour l'hydrologie urbaine par exemple.

M. CHERON. - Nous avons utilisé, quant à nous, les vitesses de $240 \mathrm{~mm} / \mathrm{h}$, mais sommes revenus à $60 \mathrm{~mm} / \mathrm{h}$. On obtient une précision correcte jusqu’à $1 / 1000$ de jour ( 1 min 24), mais cela alourdit considérablement la saisie. D'autre part, il ne faut pas négliger la difficulté de synchroniser les pluviogrammes entre cux. Cela impose de passer deux fois par semaine. Nous avons stocké de cette façon sur support informatique 200 annéesstations pour des bassins-versants expérimentaux.

M. GUILLOT (EDF, DTG). - EDF a passé commande en février 1980 de 300 enregistreurs à cassette au prix unitaire de $3000 \mathrm{~F}$ hors taxes.

Actuellement le nombre d'appareils de ce type en service continu est de 150 .

Le temps de lecture d'une cassette, qui peut contenir 3 mois de données 6 minutes, est de 3 min environ.

M.GALEA. - Le coût de $3000 \mathrm{~F}$ de l'enregistreur DTG est, semble-t-il, réservé à EDF et quelques autres organismes.

$M$. GROSSE (Bureau de l'Eau). - Nous avons en cours de dépouillement les données pluviographiques de Strasbourg et Nancy. On envisage en 1982 le dépouillement d'une cinquantaine de stations synoptiques avec une table de digitalisation.

M. CAZENAVE. - Un séminaire sur la mesure est organisé à Lyon bientôt. Il serait intéressant à cette occasion que toutes les expériences qui ont été faites puissent être confrontées, qu'elles concernent la pluic ou les débits, car le problème d'enregistrement est fondamentalcment le même.

Le Président. - Un besoin d'harmonisation se fait sentir afin, d'une part, d'obtenir des matériels moins coûteux, par une standardisation accrue, et, d'autre part, de développer des réseaux répondant aux exigences de différents usagers (les hydrologues, bien sûr, mais aussi les agronomes, les personnes chargées de l'aménagement du territoire, et les météorologistes).

La question de la coordination de lautomatisation des réscaux climatologiques, et singuliérement pluviométriques, retient toute l'attention du Conscil Supérieur de la métćorologic aux travaux duquel participent : hydrologues agronomes, représentants des services publics ... etc.

Les matériels anciens existants doivent continuer à être exploités, mais dans les cas, nombreux aujourd'hui, de réseaux nouveaux, les méthodes nouvelles évoquées trouvent lcur utilité, d'autant que le coût des moyens de transmission est lui-même élevé. Un compromis doit souvent être trouvé entre la sécurité de l'information et les coûts. Je remercie M. GALEA de sa communication, ainsi que ceux qui ont participé à la discussion. 


\section{Abstract \\ Direct acquisition of rainfall data on site}

Rainfall readings convey a great deal of information, but their execution requires manual or semi-automatic recording (digtalizing table) and a substantial amount of time which is often incompatible with available staff resources. To automatise the discretisation phase, coding of rainfall conditions directly on-site on magnetic cassette tape has been undertaken using off-line coders.

The magnetic recorder of precipitation takes the form of a box $(33 \mathrm{~cm} \times 22 \mathrm{~cm} \times 20 \mathrm{~cm})$; it is a multi-channel device (6) for digital and analogue inputs. Its quite flexible basic layout makes it possible to adapt the six input channels to the user's requirements according to the parameters to be recorded. Our purpose being to record rainfall conditions, a layout has been selected in the light of ECMA norms 341 to obtain the greatest possible autonomy and the most reliability in reading the magnetic cassette tapes. This layout corresponds to a single digital input channel served by the six electronic meters of the six "strappe" channels; observation time is six minutes and each record contains 26 bytes.

The operating principle is the cumulation in each meter (6) of the impulses generated during six minutes at each tilt of the cup. After 36 minutes the contents of the meters are transferred in a single block to magnetic storage. The logic of the transfert to tape increments the various blocks progressively by one unit.

Data processing involves the use of a cassette deck and software designed on TEKTRONIX $16 \mathrm{~K}$ (read-in) and on IRIS 80 (processing), which are themselves integrated in the basic processing programmes on hydrology and, therefore, articula ted with the subsequent automatic processing.

This coder-recorder system, which meets the various requirements, is in conformity with the layout described above. The system meets the following conditions as regards the acquisition and processing of the rainfall for the experimental catchment basic of the Orgeval:

- environment conditions $\left(-75^{\circ} \mathrm{C}<\mathrm{T}^{\circ} \mathrm{C}<40^{\circ} \mathrm{C}\right)$;

- autonomy (power source for three months, cassette recording for 2.5 months) :

- observation time (6 minutes), which yields good knowledge of rainfall conditions over shorter periods (seven observation stretches of two seconds to sixty minutes):

- (negligible) time drift which guarantees adequate synchronization of rainfall events acquired by the various sensors $c$ a given dense system. 